\title{
Perceptions, Experiences, and Needs of Parents of Culturally and Linguistically Diverse Children with Autism: a Scoping Review
}

\author{
Despina Papoudi ${ }^{1}$ (D) Clara Rübner Jørgensen ${ }^{1} \cdot$ Karen Guldberg $^{1} \cdot$ Hedda Meadan $^{2}$
}

Received: 28 December 2019 / Accepted: 20 May 2020 / Published online: 16 June 2020

(C) The Author(s) 2020

\begin{abstract}
A scoping review of studies carried out in the UK and the USA was conducted to explore the perceptions, experiences, and needs of culturally and linguistically diverse families of children with autism. Overall, 32 articles met the inclusion criteria, 25 studies were conducted in the USA and 7 studies in the UK. Four themes emerged including (a) knowledge and beliefs about autism and their impact on the family; (b) autism and family life; (c) family experiences of accessing services and support, and parents' needs; and (d) multilanguage. Findings highlighted that family perceptions of autism are influenced by an often-reported lack of knowledge; experiences include social stigma as well as difficulties in having access to services. Needs indicate the development of culturally sensitive interventions, information in multiple languages, and parent-professional collaboration. Implications for research, policy, and practice are included.
\end{abstract}

Keywords Autism $\cdot$ Parents $\cdot$ Bilingualism $\cdot$ Multilingualism $\cdot$ Culture $\cdot$ Ethnicity

\section{Introduction}

Autism affects how a person acts, communicates, learns, and interacts with others. The global prevalence of autism is rising with an estimated worldwide prevalence of $1-2 \%$ (Baxter et al. 2015). While the prevalence rate has been reported as 1 in 59 children in the USA (Baio et al. 2018) and approximately $1 \%$ of the population in the UK (Baird et al. 2006) there is considerable global variation in prevalence rates (Elsabbagh et al. 2012). In addition, there are significant

Despina Papoudi

d.papoudi@bham.ac.uk

Clara Rübner Jørgensen

c.joergensen@bham.ac.uk

Karen Guldberg

k.k.guldberg@bham.ac.uk

Hedda Meadan

meadan@illinois.edu

1 School of Education, Disability Inclusion and Special Needs (DISN), University of Birmingham, 52 Pritchatts Rd, Birmingham B15 2SA, UK

2 Department of Special Education, University of Illinois, 1310 South Sixth Street, Champaign, IL 61820, USA differences within countries with regard to autism diagnosis and access to services, with minority ethnic groups from lowincome families, in particular, being underrepresented among children who are diagnosed with autism. For example, in the UK, Dockrell et al. (2014) reported that the prevalence of autism in children of Asian heritage is half of the prevalence in white British students. Similarly, studies from the USA have found that non-white children were less likely than white children to have received a formal autism diagnosis (Mandell et al. 2009) and that they tend to be diagnosed much later (Mandell et al. 2002).

Culturally and linguistically diverse families are also likely to find it more difficult to access the information, help, and resources they need and to navigate health and education systems (Lim et al. 2018). Studies have reported scepticism around services and how confusing these can be to access (Fox et al. 2017; Hussein et al. 2019). In addition, Latino and African American children in the USA tend to receive fewer intervention services than white children with an autism diagnosis (Magaña et al. 2013; Pearson and Meadan 2018). Angell and Solomon (2017) argue that disparities are caused by a 'cultural deficit' discourse, assuming that Latino parents do not have the knowledge or ability to fight for services. Latino families often face resistance from professionals to care for their children (Angell and Solomon 2017). 
Paradoxically, research has also shown that rates of autism are higher in some ethnically diverse populations (Lehti et al. 2013; Bolton et al. 2014) and that those families may be more likely to have a child with autism and a learning disability (Bolton et al. 2014). Despite this, there are significant gaps in understanding how different cultures and beliefs influence the unique health and educational needs of children with autism from diverse backgrounds (Davenport et al. 2018). Furthermore, autism can be constructed differently in different cultures and there is a need to develop a better understanding of cultural orientations towards autism. In Nicaragua, for example, autism is not defined or acknowledged (Kim 2012). Somalis living in North America and the UK have attributed the term 'Western disease' to autism because there is no word for autism in their native language, and many people in the Somali community believe that autism does not exist in Somalia (Decoteau 2017). A UK study of parents of children with autism from Somalia found that cultural attitudes and language differences were factors that influenced their understanding and acceptance of their child's difficulties (Fox et al. 2017). A recently published study (Kinnear et al. 2016) with a sample of 502 families from USA and Canada showed that parents report that they experience stigma and that this adds to the difficulty of raising a child with autism. In addition, experiences of stigma related to having a child with autism can lead to social isolation and exclusion (Kinnear et al. 2016) and to mental health difficulties (Papadopoulos et al. 2019).

Research on the needs of culturally and linguistically diverse children with autism and their families has found that minority ethnic populations are underrepresented in research studies (Centers for Disease Control and Prevention 2014; Mandell et al. 2009; Perepa 2014). Current understandings of autism are thus primarily based on research conducted with white participants (Hoekstra et al. 2018). As a consequence, there is little understanding of how different cultures and beliefs influence the needs of families from diverse backgrounds (Davenport et al. 2018). This, in combination with the fact that few studies have emerged from the Global South, indicates that the knowledge produced often reflects specific types of culture and communities. This presents a gap in the literature, which indicates the importance of capturing the complexity of life for people with multiple marginalized identities and for understanding how different power structures interact in the lives of minority ethnic families with children with autism.

Addressing this gap, the present paper focuses on the perceptions, experiences, and needs of culturally and linguistically diverse (CLD) families of children with autism in the USA and the UK. The term CLD used in the paper broadly refers to individuals whose native language is not English and/or cultural background is not white British or European American. The term reflects an intersectional approach as some of these families have unique multiple characteristics including socioeconomic status (SES), geographic setting, and personal situation (e.g., migrant or refugee status). Burke and Goldman (2018) have defined CLD as 'individuals who do not belong to mainstream culture because of their identity with a minority race, ethnicity or linguistic group' (p. 4). By adapting a terminology around CLD rather than ethnicity (as is usually the case in the UK) or 'race' (which is often used in the literature in the USA) we account for differences and similarities between the two countries under study. A wide variety of characteristics and terminologies are used in the autism field. In this paper, we use the terms 'autistic individuals, children, and young people' to include all those who have a diagnosis of autism or any other autism spectrum condition. The term 'disorder' is not used though, other than when directly quoting the work of people who have used this term.

\section{Method}

\section{Study Selection Process}

The article selection process for this scoping review followed the strategy outlined by Arksey and O'Malley (2005) and the Preferred Reporting Items for Systematic Reviews and MetaAnalyses (PRISMA) framework (Moher et al. 2009). Empirical studies that met all of the following inclusion criteria were included in the review: (a) focused on individuals, 18 and younger, with autism (e.g., autism spectrum disorders, pervasive developmental disorders); (b) focused on the experiences and perceptions of CLD families (e.g., minority groups, migrant, immigrant, refugees); (c) published in a peerreviewed journal, in English, between 2000 and 2018; and (d) conducted in the USA or the UK. Articles were excluded if they only focused on (a) screening and diagnosis tools; (b) economic costs of autism; (c) validation of instruments, genetic testing, medication, and risk factors; and (d) methodological issues related to recruitment.

A keyword search was performed in four databases: (1) PROQUEST Social Sciences Premium Collection (Education Collection), (2) EBSCO ERIC, (3) PSYCINFO, and (4) SCOPUS including MEDLINE. The search algorithm included all possible combinations of keywords from the following three groups: (a) autism, autism spectrum disorders, pervasive developmental disorders, or autistic disorder; (b) culturally and linguistically diverse, bilingualism, multilingualism, English language learner, English as additional language, ethnicity, race, migrant, immigrant, or refugees; and (c) family, parents, mother, or father.

Titles and abstracts of the articles identified through the keyword search were screened based on the study selection criteria. Potentially relevant articles were retrieved to evaluate the full text. A cited reference search (i.e., forward reference search) and a reference list search (i.e., backward reference search) were conducted using the relevant articles that met 
the selection criteria. Next, the articles identified through the forward/backward reference search were further evaluated and selected using the same selection criteria. A repeated reference search on all newly identified articles was conducted until no additional relevant articles were found.

A total of 1278 sources of references and abstracts (records identified through database searching, $n=1267$, and records identified through other sources, $n=11$ ) were found with the use of the search terms. These sources were generated in an Excel spreadsheet. They were reviewed and filtered by relevance and the focus of the study (Table 1). Three hundred seventy-one were duplicates and were removed; the remaining
907 records were carefully screened to double check for duplicates and filtered by relevance and the focus of the study. Six hundred twenty-seven records were excluded because they did not meet the inclusion criteria. The remaining 280 records were reviewed, and the authors sought to solely identify studies that examined the experiences, perceptions, and needs of CLD families. Two hundred thirty-nine records were further excluded, and as a result of this process 41 papers passed the title and abstract phase and were identified as eligible for inclusion. An inter-rater reliability check was conducted to confirm that all included studies met the inclusion criteria and addressed the aims of the scoping review. All

Table 1 PRISMA 2009 flow diagram

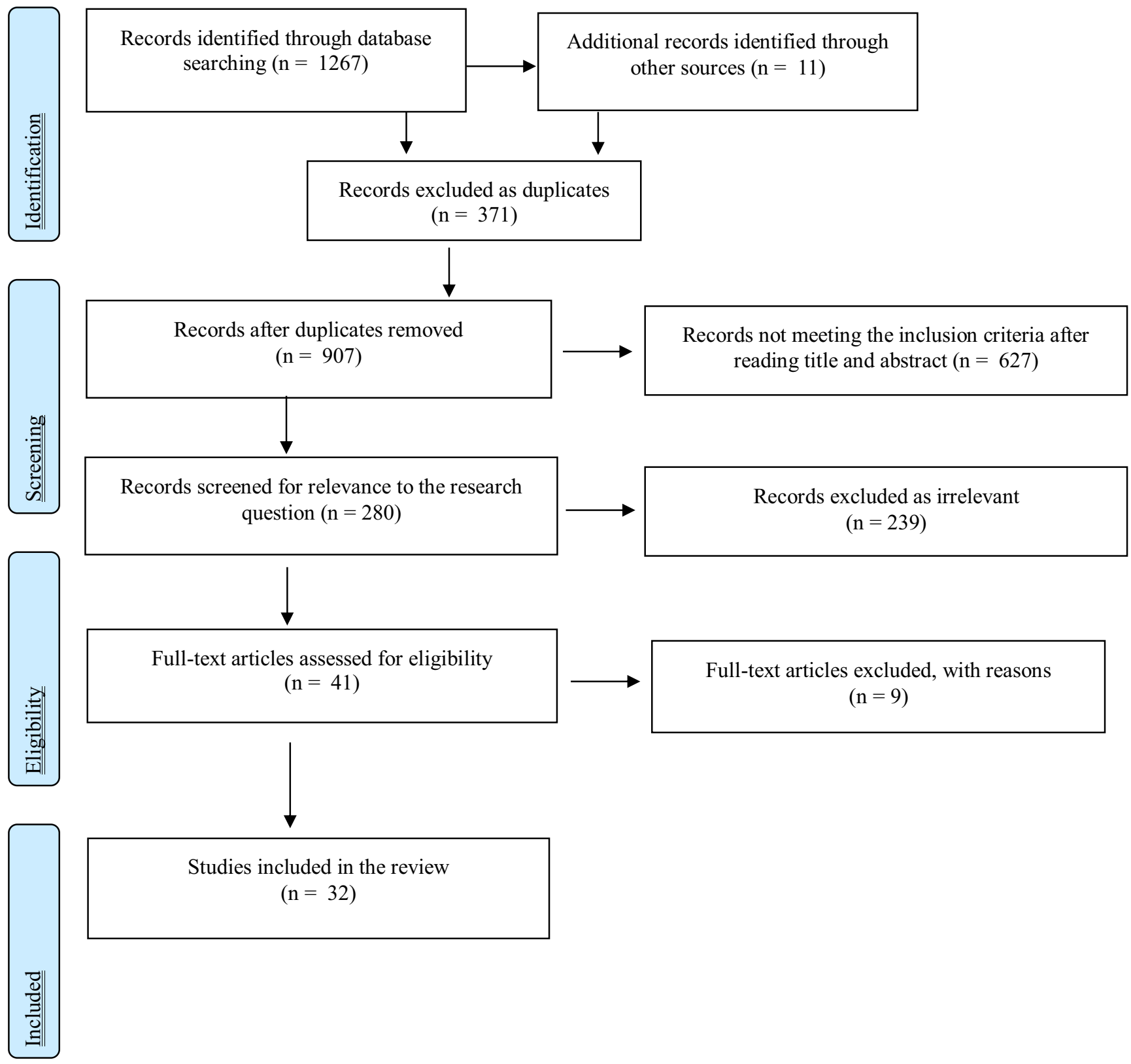


ambiguities were discussed, and the inter-rater reliability resulted in $97 \%$ rating agreement. Full text of papers that passed the title and abstract phase were downloaded and screened independently. All authors reviewed the full papers and further excluded 9 studies with a reason. This process resulted in the final inclusion of the 32 studies.

A standardized data extraction form was used to collect the following methodological and outcome variables from each included study: (a) author(s) and publication year, (b) country where a study was conducted (USA or UK), (c) purpose of the study, (d) individuals with autism (number, age, gender, diagnosis), (e) family members (number, gender, role in the family), (f) diversity characteristics (e.g., socioeconomic group, ethnicity, religion, refugees/immigrants status), (g) research design and methodology, and (h) key findings. An inter-rater reliability check was conducted for the standardized data extraction form on the basis of 5 papers by two graduate students, one of whom was based in the USA and one of whom was based in the UK. The students blindly reviewed the same papers, and the matrix was revised to ensure appropriate categorizations, consistency, and credibility of the data. A second inter-rater reliability check of $25 \%$ of all papers was conducted after the full completion of the matrix. This reached $100 \%$ agreement. The data from the matrix were used to report the results, and qualitative content analysis was applied to organize the literature under key themes and sub-themes.

\section{Results}

The majority of the identified 32 studies were published between 2016 and $2018(n=19)$, followed by studies published between 2010 and $2015(n=12)$. One study was published between 2000 and $2009(n=1)$. The majority of the studies were conducted in the USA $(n=25)$, while seven studies were conducted in the UK. A total of 5602 parents (81 fathers and 330 mothers), seven other family members, and a total of 5363 individuals with autism participated across 27 qualitative studies (e.g., ethnography, case study, interview, narrative inquiry), one quantitative (survey) study, three mixed methods studies, and one study with unspecified method. The sample size of participants is skewed by the paper by Benevides et al. (2016) in which they report the findings of statistical analysis within data sets from the National Survey of Children With Special Health Care Needs in the USA. This sample included 5178 participants, and no information about the gender of the parents was provided.

In relation to the sample of the family members, mothers and fathers participated in 13 studies, only mothers participated in 9 of the studies, only fathers in 2 of the studies, and parents and other family members (e.g., siblings, cousin, grandmother) participated in 5 studies. In 3 studies the gender of the parents was not specified. In terms of ethnicity and cultural background, participants described themselves as 'African American' in 2 studies, 'Afro-Caribbean' in 1 study, 'Asian American' in 3 studies, 'Asian Indian' in 1 study, 'Black' in 3 studies, 'Chinese' in 1 study, 'Hispanic' in 2 studies, 'Korean' in 1 study, 'Latino/a' in 2 studies, 'Mexican' in 2 studies, 'South Asian' in 5 studies, and 'Somali' in 5 studies, and in 4 studies the ethnicity was not specified. In 16 studies parents identified themselves as immigrants and in 2 studies as migrants.

The range of the sample with the individuals with autism ranged from 1 to 10 ( $n=10$ studies), 20-60 ( $n=3$ studies), and in one study the sample size was 5178 (Benevides et al. 2016). The individuals with autism ranged in age from 0 to 23 years old, and the majority were under 18 years old. In relation to gender, the individuals were boys and girls in 11 studies, boys in 12 studies and only girls in 1 study, while in 8 studies the gender was not specified. In relation to autism diagnosis, parents reported the diagnosis of their children as 'ASD', 'autism', 'Autistic disorder', 'Asperger's Syndrome', 'High-functioning autism', 'PDD', or 'PDD-NOS'.

Following the extraction of the data, all authors read the full papers. The authors identified and discussed initial themes related specifically to the purpose of the review. Coding consistency was ensured by all authors agreeing on the themes and by one author completing a second round of coding. Four key themes were identified in the analysis of the 32 papers included in this scoping review: (a) knowledge and beliefs about autism and their impact on the family; (b) autism and family life; (c) family experiences of accessing services and support, and parents' needs, and (d) multilanguage. These broad themes were furthermore divided into a number of sub-themes (see Table 2).

\section{Theme 1: Knowledge and Beliefs About Autism and Their Impact on the Family}

Lack of Knowledge Among Parents and in the Community A common theme in many of the papers was a lack of knowledge among parents about autism prior to their children's diagnosis (e.g., Fox et al. 2017; Ijalba 2016). In the USA, a quantitative study of parent knowledge and perceptions of autism across ethnic groups, Ratto et al. (2016) found that Latina mothers had significantly less knowledge of autism than white mothers, even after controlling for education. Huang and Zhou (2016) also reported that out of the Chinese families they interviewed in their USA study, almost all viewed autism as 'a temporary and transient state of being that reflected delays in a particular developmental stage, rather than a disorder that causes global and far-reaching delays in the child's cognitive, adaptive, and social-emotional development' (p. 64). Parents furthermore found symptoms confusing and were uncertain about the implications of the condition. Confusion about autism was discussed by Selman et al. 
Table 2 Key themes and sub-themes emerging from the scoping review

\begin{tabular}{|c|c|}
\hline Key themes & Sub-themes \\
\hline 1. Knowledge and beliefs about ASD and their impact on the family & $\begin{array}{l}\text { a. Lack of knowledge among parents and in the community } \\
\text { b. Explanatory models } \\
\text { c. The impact of beliefs on parents' actions and coping }\end{array}$ \\
\hline 2. Autism and family life & $\begin{array}{l}\text { a. Stages of acceptance } \\
\text { b. Family dynamics and the involvement of extended family }\end{array}$ \\
\hline $\begin{array}{l}\text { 3. Family experiences of accessing services and support, } \\
\text { and parents' needs }\end{array}$ & $\begin{array}{l}\text { a. Access to support } \\
\text { b. Experiences of the services provided. } \\
\text { c. Support groups and information about culturally diverse families with autism } \\
\text { d. Parents' needs }\end{array}$ \\
\hline 4. Multilanguage & $\begin{array}{l}\text { a. Family perceptions of multi-language } \\
\text { b. Families views about professional perceptions of multi-language }\end{array}$ \\
\hline
\end{tabular}

(2018) and Fox et al. (2017) in their study of UK Somali families, who struggled to categorize and understand autism, often noting the perception that autism does not exist in Somalia. The parents received conflicting messages from their own community, who also sometimes suspected that the parents themselves had caused their children's autism. This combined with the general lack of understanding of autism in the community and stigma around disability and mental illness led to frequent stereotyping and othering of parents of children with autism.

Lack of knowledge of autism in the community, resulting in criticism of parents for having caused the condition, and subsequent experiences of isolation and stigma were described in several papers and in relation to a range of CLD communities, including the UK Somali community (Fox et al. 2017; Hussein et al. 2019), the African American community (Burkett et al. 2015; Lovelace et al. 2018), African Immigrant mothers in the UK (Munroe et al. 2016), Asian Indian families in the USA (Zechella and Raval 2016), and Hispanic families in the USA (Cohen and Miguel 2018; DuBay et al. 2018; Ijalba 2016). However, these sometimes coexisted with other responses. DuBay et al. (2018) for example described that while some extended families rejected the diagnosis, others were very supportive. Similarly, Fox et al. (2017) reported that while some of the UK Somali parents in their study avoided contact with neighbours and friends, others talked openly and with pride about their children, and this was to some extent related to their own stage of acceptance and concerns about stigma. The lack of understanding and awareness of autism in the UK Somali community was described in Hussein et al. (2019) as a reason for parents being insulted by family and community members. However, at the same time, some parents rejected this and refused to hide their children from the community.

Explanatory Models When trying to understand the cause of their children's autism, families drew on a range of religious or cultural explanations. This was common across several of the communities described in the papers. Ijalba's (2016) study of Hispanic immigrant mothers identified traumatic events, unexplained fear, and the mothers' own sadness during their pregnancy as common explanatory factors, and these were particularly prevalent among mothers who did not have legal permanent residency or lacked support from extended family. Ijalba (2015) also described other external factors perceived by Hispanic mothers to have caused autism, including Quechua traditional beliefs about wind sickness and Latin American folk beliefs about fright and hurtful gazes.

In Jegatheesan (2011) and Jegatheesan et al. (2010a, 2010b) study of South Asian Muslim immigrants in the USA, parents interpreted the meaning of having and raising a child with autism through their faith, and described their child with autism as a gift from Allah, and they themselves as 'chosen' for the task of raising the child. Similarly, the UK Somali parents in Hussein et al.'s (2019) study interpreted their child's autism as the will of Allah and as a test that they would later be rewarded for having accomplished. In addition, some of them believed that autism was the result of a jinn entering the child's body.

The Asian American immigrant parents in Wang and West's (2016) study also invoked a range of explanations when trying to find the cause for their children's autism, for example having cooked baby food in the microwave, the child suffering a fall where 'Chi' had been leaked, or the mother violating a pregnancy taboo. In addition, mothers cited environmental and lifestyle factors and fathers' diet, genetic factors, the environment, vaccine injections, and modern technology. Vaccines were also mentioned by the parents in Hussein et al. (2019) and Cohen and Miguel (2018) as a possible cause of autism, with the former also mentioning a lack of sun and vitamin D and the latter genes and family history. The idea that autism was related to maternal behaviour during pregnancy was common in the studies. The families of two of the African American mothers in Lovelace et al.'s (2018) 
study had blamed their children's autism on the mothers taking drugs or drinking while pregnant, although the mothers denied this. Gilligan's (2013) study of South Asian Muslim mothers in Bradford, UK, had been told by their networks that autism derived from their behaviour or diet during pregnancy. Similarly, Jegatheesan et al.'s (2010a) Somali parents in the USA both searched for a medical diagnosis and listened to their relatives, who explained the children's autism with the mother's health or the consumption of prohibited foods during pregnancy and a lack of adherence to religious practices.

As this shows, participants provided a range of explanatory factors, some based on their faith or spiritual convictions, and others on their beliefs about the environment and their own behaviours. In some cases, parents differed from the extended family and community in their explanatory models, as described above by Lovelace et al. (2018), and sometimes parents invoked different models simultaneously. Munroe et al. (2016) for example showed that the three African immigrant mothers in her UK study to some degree drew upon African cultural beliefs, but also 'appeared to disagree with the majority of these' (p. 811). The mother who had most recently migrated particularly worried that autism had been caused by a curse and sent money to Africa for 'sacrifices' to stop it. However, the mothers generally rejected the common perception in the African community that their sons were 'mad', 'possessed', or naughty and adhered to the 'new explanation' - autism, which appeared to protect them from feeling helpless and gave them some power over their situation. Nevertheless, the contradictions between this model and the traditional African beliefs of their community led to painful conflicts within their cultural identity and feelings of marginalization from both cultures.

The Impact of Beliefs on Parents' Actions and Coping In addition to identifying lack of knowledge and a range of beliefs about autism, the papers showed a strong link between the parents' knowledge and beliefs and their actions. For example, Luong et al. (2009) described how South Asian parents in the USA relied on faith to cope with their children's condition and used religious practice to provide them with hope and sense of perspective. Jegatheesan et al. (2010a) mentioned that parents drew on elders in their choice and use of folk treatments and furthermore believed that their child's condition could be stabilized by religious and dietary practices. Jegatheesan (2011) also found that parents drew on their religion when arguing for a full inclusion of the children in family and community activities.

Parental beliefs about disability held by the Hispanic mothers in Ijalba's (2015) study also influenced how they engaged with and participated in their children's education. One mother, for example, kept her child home from a school summer programme; another often kept her daughter home from school all together, as she believed the protective environment at home was better for her. Finally, Huang and Zhou
(2016) showed that the level of difficulty and stress experienced by the Chinese parents in their study depended on the parents' level of acceptance of their child's diagnosis, with those who had low acceptance levels experiencing more difficulties with the demands of their child. This shows the importance of acknowledging the processual nature of family approaches and the diverse impact of autism on family lives.

\section{Theme 2: Autism and Family Life}

Stages of Acceptance Many of the families discussed in the papers described a sense of shock when their children received a diagnosis of autism (e.g., Baker 2017; Jegatheesan et al. 2010a), with some experiencing significant subsequent isolation (Huang and Zhou 2016; Munroe et al. 2016). Fox et al. (2017) noted that many of their participants were unfamiliar with the word autism and therefore were left feeling confused upon getting the diagnosis. Illustrating the dynamic nature of family responses to autism, Luong et al. (2009) identified a range of phases, which their Southeast Asian families went through. This included denial and passive coping, empowerment, redirecting energy, shifting of focus, rearranging lives and relationships, changed expectations, social withdrawal, spiritual coping, and acceptance. This gradual acceptance and development of coping mechanisms was found in several of the studies. The African immigrant mothers in Munroe et al.'s (2016) study initially went through a phase of acquiring information about autism through the internet and by talking to professionals. Through this they came to an agreement with the diagnosis, which offered a framework for understanding their children and how to help them. The Korean Immigrant mothers in You and Rosenkoetter's (2014) study had found it difficult to accept their children's condition at first, but later began to adjust their maternal role to address the specific needs of their children. This included reevaluating their role as a mother (with some giving up their jobs to look after their child) and their priorities as a family. Similarly, the Asian Indian families in Zechella and Raval's (2016) study described how their children's disabilities had led them to change their perspectives on life in general and their particular role as parents and advocates for their children.

Parental self-sacrifice was identified as a major theme in Huang and Zhou's (2016) study of Chinese families of children with autism, and this was particularly prevalent in families with more traditional values. Wang and West (2016) also found that the Asian American Immigrant mothers in their study put their careers aside to look after their children, but not always without grievance. The mothers tended to employ emotion-focused coping and religious coping, whereas fathers tended to use rationality-focused coping strategies, e.g., searching for related knowledge and going on a journey of hope and illustrating the importance of gender-specific coping mechanisms and family dynamics. 
Family Dynamics and the Involvement of Extended Family Families varied in the extent to which they drew on extended families and community members for support, and this depended on the wider understanding of autism in the community. The South Asian Muslim immigrant families described by Jegatheesan et al. (2010a) did not view their extended families as a resource for coping with the diagnosis and refrained from openly talking about their emotions because they believed such talk could negatively impact on their extended families. Huang and Zhou (2016) linked the isolation and deterioration in social relationships experienced by several of the Chinese families in their study to their fear of 'loosing face'. Fox et al. (2017) and Selman et al. (2018) described the negative perceptions of mental illness within the Somali community as a source of social isolation and exclusion for the families, due to neighbours or friends avoiding their company and parents self-isolating to protect their children. Selman et al. (2018) furthermore described how parents engaged in a complex negotiation around the diagnosis of autism, with fathers particularly struggling to accept it. Wang and West (2016) also mentioned the particular difficulties of fathers in accepting the diagnosis, with some being in denial and therefore not telling their families about it.

Relationships between fathers and mothers were described in some of the studies, including Kim and Kim (2017) who found that linguistic barriers, stereotyping, and contentious gender roles led to marital conflicts and instability, as the Korean immigrant mothers they studied struggled to raise their children with disabilities by themselves at home. Conversely, Zechella and Raval's (2016) Asian Indian families considered their relationships to have been strengthened by them focusing on their child rather than their own differences. Finally, the relationship with siblings was discussed in a few of the papers, most significantly in Sage and Jegatheesan (2010) who presented two contrasting examples of how a European American and Asian American family explained autism to their typically developing sibling and how this consequently impacted on the siblings understanding the interactions between the two.

\section{Theme 3: Family Experiences of Accessing Services and Support, and Parents' Needs}

Access to Support Hannon et al. (2018) identified three key reasons for differences in diagnoses between ethnic and racial groups: disparities in access to care, disparities in diagnosis timing, and disparities in the frequency of dual diagnosis. In relation to the second reason - timing - several of the papers reviewed mentioned that CLD groups experience delays in diagnosis and have greater difficulties obtaining care than majority ethnic families (e.g., Benevides et al. 2016; Burkett et al. 2015). One explanation for delayed diagnosis provided in the articles was the lack of knowledge of families and their beliefs about disability (Luong et al. 2009). As noted by Fox et al. (2017):

Community attitudes towards mental illness, challenging behaviours and disability, combined with the lack of vocabulary to describe and explain autism, made acceptance extremely challenging. It was therefore difficult for parents to recognise that their child's disability was characteristic of autism. These attitudes also prevented parents from sharing their concerns about their child, meaning that assessment and diagnosis were sometimes delayed (p. 309).

Furthermore, it was common for parents to approach members of their families first, rather than seek professional advice (Burkett et al. 2015; Fox et al. 2017; Luong et al. 2009), and these often advised them not to be concerned (Fox et al. 2017; Jegatheesan et al. 2010a).

Lack of familiarity with their rights as parents of a child with a disability and the system in general were also identified as reasons for delayed access to services and collaboration with professionals (e.g., Jegatheesan et al. 2010a). Fox et al. (2017) found that parents lacked familiarity with the many different systems and that this, combined with their language barriers, contributed to delays in accessing support for their child. The UK Somali parents in their study furthermore displayed a 'widespread concern that their child would be taken away from them if they were seen to be having difficulty coping with the demands of raising a child with autism' ( $p$. 312). Hussein et al. (2019) similarly described a general distrust in Western Medicine and medical explanations among Somali families in the UK but also identified a move towards combining interventions based on faith with more formal advice from health care professionals.

In addition to such family-based explanations, some of the studies acknowledged that these worked in parallel with more systemic inequalities to explain delayed diagnosis. Burkett et al. (2015) identified a combination of diagnostic bias, poor access to health care, child presentation, and family symptom interpretation as cultural influences on diagnostic delays and treatment in urban African American families. Illustrating how these factors worked in tandem, they described how the families in the study engaged in 'family protective care', practices that promoted the children's independence, treated them as 'normal' to ensure their independence and prevent them from experiencing discrimination, and taught them to be watchful. They argued that these practices in turn led to higher functional skills in African American children with autism and contributed to diagnostic delays. Potentially supporting this, Hannon (2017) mentioned that Black American children were two and a half times less likely to be diagnosed with autism on their first visit to speciality care than white American Children. 
Ratto et al. (2016) also invoked a combination of family and systemic factors to explain diagnostic delays. While their study showed no major differences in the time when Latina and low SES mothers began to develop concerns about their child, they argued that delays in diagnosis could be caused by the difficulty the mothers had in navigating the system, the way providers and health care professionals responded to them, and that the mothers described their concerns differently than white mothers, resulting in professionals not considering autism as a potential diagnosis. Finally, Benevides et al. (2016) found that families from different ethnic groups varied in their perception of treatment needs, with caregivers of minority children significantly less likely than white non-Hispanic children to report a perceived need for prescription and medication, Black non-Hispanic children significantly less likely to report a need for child and family mental health care than white non-Hispanic children, and English-speaking Hispanic children and black non-Hispanic children significantly more likely than white non-Hispanic children to report a need for therapy services. Benevides et al. (2016) linked parental beliefs about the perceived need for services, their help-seeking behaviour, and the interventions that their children with autism receive, and based on this, they argued that practitioners need to be aware of the multiple reasons why families may not pursue treatments and that families from different racial and ethnic backgrounds may need additional communication and support in making treatment decisions for their children with autism.

Experiences of the Services Provided In addition to the question of accessing services, the papers also found that families from CLD groups reported a number of challenges in the services they received. The African American mothers in Lovelace et al.'s (2018) study had all experienced feeling discriminated against because of their race in one or more interactions with service providers. Communication was a key consideration in the review studies, with language barriers described as significantly impeding access to support and communication with service providers (DuBay et al. 2018; Hussein et al. 2019; Luong et al. 2009). Fox et al. (2017) noted that even proficient English speakers reported difficulties in understanding the words used by service providers. This was also found in You and Rosenkoetter's (2014) study, where the Korean American mothers identified the special needs terminology and medical jargon as barriers to their communication with educators and specialists. Jegatheesan et al. (2010a) furthermore described how the South Asian Muslim immigrant families in their study had experienced that therapists had been insensitive in their communication and interaction, by showing a lack of trust in the parents' own reporting, a lack of interest in their background, and by using unfamiliar jargon. They also reported 'cultural clashes and mismatches' in relation to the use of multiple languages in the home and the daily lifestyle of the families, which included many extended family members, friends, and neighbours. Therefore, the families expressed a preference for professionals of a similar cultural and linguistic background. In addition, Jegatheesan et al. (2010b) found that the parents felt disturbed by the professionals' emphasis on the children's deficits, which they contrasted with their own more positive vision for the future of their children.

DuBay et al. (2018) furthermore identified a difference in the experiences of families of different types of services. The Latino caregivers in their study had felt more included in individual private treatments but discouraged from being present in school-based interventions, resulting in them not learning from the strategies in the latter. The authors questioned the quality of the providers employed by different agencies and 'whether less capable or motivated providers are at times assigned to Latino Spanish-speaking families whose limited English fluency may prohibit formal complaint' (p. 1636). Further acknowledging inequalities in access to quality services, Lovelace et al. (2018) mentions that in the US context 'nice programmes' are often in the suburban areas and that urban families have difficulty in accessing them. Gilligan (2013) also calls for recognition that 'in Britain and elsewhere, support groups for parents may be clustered in white, middleclass areas and may offer services that many ethnic minority parents will find difficult to access' (p. 405).

In summary, the current research on the experience of CLD families of children with autism highlighted that these families need intervention programmes that are sensitive to linguistic and cultural diversity and that provide information that is appropriate for culturally and linguistically diverse groups. The families needed practitioners to take into account the lack of bilingual and culturally sensitive programmes, to review the information people may be getting in their communities (Lovelace et al. 2018), the stigma they may experience (Munroe et al. 2016), their cultural and religious explanatory models and values (Cohen and Miguel 2018; Gilligan 2013; Hussein et al. 2019; Ijalba 2015), and coping styles (Huang and Zhou 2016). It also appears that there is a need for a more individualized approach because while clinicians and professionals were urged to take culture into account and develop programmes with the input of cultural communities (Munroe et al. 2016), it was also noted that some parents expressed willingness to set aside their cultural beliefs, if they thought this would benefit their children (DuBay et al. 2018; Perepa 2014), as clearly stated by a mother in DuBay et al. (2018) study: 'I'm practical. If I have to leave my culture aside, I will leave it, my son is first... My son needs to learn that, so I'm teaching my son to point... My children come first before my culture' (p. 1630).

Support Groups and Information About Culturally Diverse Families with Autism Several of the papers mentioned the benefits of support groups consisting of other parents and families with autism (Hannon et al. 2018; Zechella and 
Raval 2016). Selman et al.'s (2018) and Fox et al.'s (2017) UK Somali families identified structured training courses on autism and parent support groups especially supportive. DuBay et al. (2018) furthermore mentioned that support groups were particularly important when the community was perceived as non-supportive, as in the case of some of the Hispanic migrant families in their study, who avoided sharing their children's diagnosis with family members and sought social support elsewhere, including support groups with other Latino families of children with autism.

The parents in Jegatheesan et al.'s (2010a) study suggested that support for families could be provided by cultural community centres that are 'actively involved in ensuring the wellbeing of their members in the US' (p. 808). Several of them however were not comfortable with support groups, as they found them 'alien' and because they had experienced language barriers, felt stressed and embarrassed to speak in mainstream groups, and 'felt lost and knew no one' (p. 806). Mothers particularly felt uncomfortable talking about feelings in front of male strangers and expressed a preference for groups divided by gender. In addition, they had found it difficult to be in groups with people who were at different levels of their understanding of autism. Luong et al. (2009) described how South Asian Parents did not find support groups helpful either, because 'I did not want to hear about other people's problems, and I did not want to burden them with mine' (p. 227). Due to the cultural stigma around mental illness, they had felt safer at home and alone. Finally, and further illustrating the complexity of matching people with support groups, one of Lovelace et al.'s (2018) participants described feeling alienated from a support group, due to being a single mother within a group predominantly composed of married couples: 'I'm like your struggles are not my struggles at all. That's how I felt. So, I felt out of place even at support groups' (p. 10). Overall, families needed structured training courses but felt ambivalent about participation in support groups.

Parents' Needs Parents have different needs in caring for a child with autism. This includes differing needs in relation to information, family and social support, financial support, explaining to others, childcare, professional support, and community services (Bailey and Simeonsson 1988), respite care, information about support services and access to these services, social acceptance, emotional support, and funding (Hodgetts et al. 2015). In the current scoping review, Benevides et al. (2016) extensively investigated the needs of CLD families in relation to medical, therapeutic, and family support services. The findings showed that caregivers of Hispanic children reported less need for prescription medications compared with caregivers of white non-Hispanic children with autism, caregivers of black non-Hispanic children with autism reported less need for prescription medications, and for child and family mental health services than caregivers of white non-Hispanic children. English-speaking Hispanic caregivers and black non-Hispanic caregivers reported greater need for occupational, speech, and physical therapy than white non-Hispanic caregivers, and no racial or ethnic differences were found in perceived need for specialty medical care or respite care. Therefore, they concluded that caregivers of children with autism from CLD groups may have different perceptions of the need for various types of care and these perceptions may impact on how they prioritize and seek care, independent of their child's specific needs. DuBay et al. (2018) also examined the unmet needs of Latino parents in relation to service provision. In this study, Latino parents highlighted the need to be involved to some extent in therapy sessions with their children, to have good communication with service providers, and to receive information for working with their children at home. Parents expressed a need for various types of support including class or therapy observations, hands-on practice, or group workshops for families. In relation to service provision, Latino parents expressed the need to work with bilingual or multilingual service providers who have professional skills and are highly motivated to have good and frequent communication with them, who are willing to build relationships and provide parental support. Similarly, parents from the Somali community in the UK also expressed the need for good communication with the child's school and assistance with language to overcome the language barrier as well as the need for support to access the education and social services (Fox et al. 2017) and the need to work in partnership with practitioners who are well informed about bilingualism, second language learning, and maintenance of heritage language (Yu 2013). In addition, Chinese parents also expressed the need to have access to interventions, which are available in their native language ( $\mathrm{Yu}$ 2013).

\section{Theme 4: Multilanguage}

Family Perceptions of Multilanguage Families generally found that learning two or more languages was important for their children with autism, as this was seen as a key way to maintain their heritage, religion, community, or family relations (Jegatheesan 2011; Kim and Roberti 2014). Jegatheesan et al.'s (2010b) study participants stated that being able to speak their native language was important for communicating with non-English-speaking family members, for example grandparents who often provided child care, and for being part of the family's social life, through which the children learned important cultural values. Full inclusion in the family was not considered to be accomplishable without immersion in multiple languages. Yu (2013) found that bilingual Chinese/ English-speaking immigrant mothers of children with autism described Chinese language as important for preserving their heritage and cultural identity, to instil pride, and to help the 
children gain an advantage on the global job market. In addition, they expressed that they would feel 'some degree of loss if the children never learned it' (p. 16). The last element illustrates the emotional element of language, which was described in several of the reviewed studies, with parents stating that they preferred their native language when showing affection and singing to their children (Baker 2017; Hampton et al. 2017). Garcia y et al. (2012) parents described speaking only in English as a great loss to the families, resulting in emotional distancing, being lost for words, and detachment from extended family and community.

Despite the benefits of learning multiple languages as described by the families, the loss experienced when speaking only English, and the lack of research to support the case for a one-language only policy, many of the parents worried that learning more than one language would delay their children's speech (Hampton et al. 2017), confuse the children (Baker 2017; Hampton et al. 2017; Ijalba 2015; Yu 2013), or negatively interact with autism traits, such as not reacting well to changes or transitions (Hampton et al. 2017). In one study, some parents however also thought that bilingualism provided opportunities to foster abilities that children with autism might find difficult, suggesting that much depended on the general verbal skills and level of functioning of the children (Hampton et al. 2017).

Due to the concerns about multilanguage being confusing for children with autism, the majority of the participants in Baker's (2017) study supported the idea behind a onelanguage approach, and other studies similarly described families adopting an English-only approach. In Yu's (2013) study for example this was described as an instrumental approach: 'A clear priority for all of the parents in the study was to address the perceived life barriers that they associated with the autistic condition. If the heritage language was perceived to be an obstacle to that goal, then it was minimized or dropped' (p. 16).

\section{Families Views About Professional Perceptions of}

Multilanguage The confusion and apparent contradictions in parents' perception of multilingualism was to a large degree related to the lack of guidance from professionals on the matter (Baker 2017), or their direct advice to raise the children mono-lingually (Hampton et al. 2017; Jegatheesan et al. 2010a; Garcia y et al. 2012) even when the parents themselves were not proficient in English. In Ijalba's (2016) study of 22 Hispanic Immigrant mothers, 13 families had been advised to shift to English only, even though 11 of them had at least one parent who was not able to communicate in English and who was the primary caregiver. Jegatheesan (2011) found that professionals often considered the children's interactions with many relatives and community members, who were often not proficient in English, 'detrimental to the child's development', and warned of the negative impact of using more than one language. This in turn led to parents feeling anxious about professionals finding out about their use of multiple languages at home.

$\mathrm{Yu}$ (2013) similarly reported that the most frequently heard advice reported by the Chinese/English speaking immigrant mothers in her study after their child had been diagnosed with autism was to start speaking English with the children as soon as possible. Only two of the parents in the study said they were told positive things about bilingualism and actively encouraged to foster heritage language learning in their children. All of the parents who were advised to speak bilingually were also told to clearly separate the use of the two languages with their children, a practice which is questioned by $\mathrm{Yu}$ (2016) as being at odds with the hybrid language practices used by families. In contrast to this, Hampton et al. (2017) found that a substantial amount of the bilingual parents of children with autism in their UK study were told that bilingualism did not present any problem, leading the authors to conclude that: 'It may be that, in the United Kingdom at least, attitudes of professionals toward bilingualism are becoming more favourable' (p. 444).

Nevertheless, the papers documented a general lack of interventions and practice material in other languages, and this also led families to choose English for pragmatic reasons (Hampton et al. 2017; Yu 2013). In general, families adopted different strategies depending on the advice they had been given, their own beliefs and their children's situation. Some differences were identified between parents, for example with parents of children who struggled with language being more worried and with individuals feeling different towards language at different times and under different circumstances. This emphasizes the importance of considering language in context and of acknowledging the broad variety of children with autism, making generic recommendations difficult (Baker 2017).

\section{Discussion}

Overall, the findings indicate that family perceptions of autism are influenced by an often-reported lack of knowledge about autism and the cultural, linguistic, and religious context in which the families find themselves. Experiences reported in the literature included initial difficulties in coping with an autism diagnosis, social stigma in the community and subsequent isolation, difficulties in accessing appropriate services, and challenges in relation to language preferences and recommendations in bilingual families. The findings indicate the need to carefully develop culturally sensitive interventions and information, and strengthening social support, taking into consideration that CLD groups are highly diverse and therefore may not all have the same support needs. 


\section{Lack of Knowledge}

Lack of knowledge about autism among CLD parents in the USA and in the UK is often associated with misconceptions of autism (Huang and Zhou 2016), which relates to a deficit model of disability (Fox et al. 2017; Ijalba 2015; Selman et al. 2018). This may be based on viewing autism as an impairment and sometimes as a mental illness (Luong et al. 2009). Parents who are unfamiliar with the notion of autism as a neurodevelopmental disorder furthermore draw on explanatory models based on the cultural beliefs of their community and on their own beliefs. These views might delay seeking advice for the diagnosis of autism and for support services (Burkett et al. 2015). However, the literature also shows that CLD parents do not always uncritically adopt the cultural beliefs of their communities and, in fact, often challenge and adopt these to fit their particular situation (Lovelace et al. 2018; Munroe et al. 2016). This illustrates the importance of not assuming that parents will necessarily adhere to particular ideas and avoiding cultural deficit approaches. It also highlights the importance of developing information material in different languages to raise awareness and knowledge about autism within CLD communities, helping parents seek earlier diagnosis and accessing culturally appropriate support mechanisms if experiencing stigmatization or isolation from their communities.

\section{Stages of Acceptance and Stigma}

The diagnostic process is a key stressor for parents, and this has been reported in studies with non-CLD families and also in the present scoping review. Overall, increased parenting stress is found in parents of children with autism prior to diagnosis and increased parenting stress has been related to less collaboration with professionals in non-CLD groups (Moh and Magiati 2012). In addition, delays in diagnosis were reported in several of the reviewed studies and careful consideration is needed as this is an important issue within autism services and is a cause of parental stress. A large-scale survey was recently carried out in the UK examining parents' experiences of autism diagnosis and a lack of cultural diversity was reported (Crane et al. 2016). In this study, a sample of 1047 parents completed an online survey, with $95 \%$ of parents describing themselves as white, and it was found that there was in average a delay of 3.5 years for receiving a confirmation of autism diagnosis, that $66 \%$ of the sample reported dissatisfaction with the manner the diagnostic process was handled by the professional and $61 \%$ were dissatisfied with the information provided post-diagnosis (Crane et al. 2016). Similarly, Hennel et al. (2016) in Australia in a large-scale study of 404 families revealed that $98 \%$ of parents viewed the information about the meaning of the autism diagnosis and accessing relevant support services as important to them, but this information was infrequently provided by professionals. Therefore, it was suggested that the presence of a support person during the diagnostic assessment could improve satisfaction and that the post-diagnosis period could be supported by a written information resource kit as parents might struggle with the amount and complexity of the information being conveyed to them during the disclosure of the diagnosis.

The review also showed that in addition to coping with an autism diagnosis, parents are experiencing stigma as a result of having an autistic child. Stigma experiences have been also reported in non-CLD groups, and it appears that stigma is a theme across different groups in the autism field. Papadopoulos et al. (2019) carried out a systematic literature review exploring the relationship between autism-related stigma and the mental health of caregivers across diverse sociocultural settings, including countries in East Asia, the Middle East, USA, and Australia. The review showed that stigma has a harmful impact on the caregivers' mental health including depression, anxiety, and psychological distress. Lodder et al. (2019) designed a short psycho-social support intervention to tackle issues related to self-stigma in parents of children with autism and asked the views from the autism community via an online survey of 112 participants. Respondents suggested that parents' self-esteem should be boosted and that parents would benefit from 'ready-made' phrases or information available to respond to instances of stigma from the public, other family members, and professionals. Respondents were also keen to meet other carers and share experiences. Furthermore, proponents of the neurodiversity movement (Kapp et al. 2013) argue that increased awareness of autism, viewing autism as a positive, valid, and equal but different identity, and accepting and celebrating autism may decrease stigma and encourage parents to seek diagnosis. Parents' beliefs about the causes of their child's disability are particularly important as they often inform potential decisions about the intervention approaches they seek for their child, and the expectations from their child (Ravindran and Myers 2012). Professionals need to be aware of the diverse beliefs that parents from different CLD groups might have as these have an impact on decisions parents make for their child's education and access to service delivery. There is a need for a more balanced approach in which parents understand the challenges of bringing up and caring for a child with autism as well as accepting their own child by challenging and rejecting stigma. Delivery of parent training programs and empowerment of parents will hopefully enable them to come into terms with an autism diagnosis, reject self-blame, and query religious concepts.

\section{Experiences of Services}

In many of the reviewed studies, parents reported difficulties in relation to access to support and this might be due to disparities in access to care and to lack of knowledge about 
autism and beliefs among ethnic groups and professionals too. Therefore, there is a need for professionals to be culturally sensitive and culturally aware of the practices the parents employ and be supportive so the parents can trust the professionals and talk to them freely about their practices. This could help professionals suggest strategies that are culturally sensitive but also alert the parents in case the practices they use might have a negative impact on the child (Ravindran and Myers 2012). Early autism intervention parent mediated programs with parents from diverse cultural and socioeconomic backgrounds can be effective in reducing parental stress and teaching strategies for increasing positive interactions between caregivers and autistic children at naturalistic environments (Rollins et al. 2019).

Furthermore, parents reported in the reviewed studies that professionals often used language that was difficult for them to follow (DuBay et al. 2018; Hussein et al. 2019; Luong et al. 2009) and, therefore, sufficient opportunities must be given to them to ask questions and professionals must be aware of this so they can facilitate questioning. The parents, both mothers and fathers, can experience significant anxiety, stress, and sadness during the diagnostic assessment and the disclosure of the diagnosis (Moh and Magiati 2012). Professionals can help by creating a trusting environment, a positive parentprofessional relationship, mentioning strengths as well as difficulties and conveying hope. Furthermore, parents find written information useful and in particular for understanding the diagnosis and explaining it to friends, family members, and school services (Abbott et al. 2013).

\section{Multilanguage}

Bi-lingual parents in the review studies expressed some confusion about the use of languages, as professionals often recommended that they communicated with their children in one language only. Nevertheless, the papers illustrate both emotional and practical difficulties of doing that, and that it sometimes had significant consequences for the family interaction and dynamics. Children with autism can acquire two languages (Gonzalez-Barrero and Nadig 2018), and research has shown that bilingually exposed children with autism used more communicative gestures and engaged in more imaginative play than their monolingual counterparts and that bilingualism did not negatively affect language development in young children with autism (Valicenti-McDermott et al. 2012). There was some indication that professionals in the UK were more supportive of bilingualism, but the findings clearly indicate the need for continuous professional development of practitioners to debunk myths about the damage of bilingualism in relation to autistic children and enabling them to provide effective positive guidance about the benefits of bilingualism in children with autism.
Many of the aspects discussed above might be common to many parents with disabilities and to parents of children with autism. However, the current findings suggest parents from diverse CLD groups face additional challenges, due to their engagement in complex cultural and linguistic environment. In addition, the parents of culturally and linguistically diverse individuals with autism represent a range of different intersecting identities, which are important to consider in relation to each other and the particular research setting. According to Thomas and Macnab (2019, p. 7) 'diversity' is at the heart of an intersectional perspective' and intersectionality considers identities as interacting with one another (Liasidou 2013). One key intersection described in the studies carried out in the USA was between disability and being black in attaining an autism diagnosis (Hannon 2017). In this vein, Decoteau (2017) recently proposed that race and nationality are integral to conceptualization and explanation of autism as observed in the Somali community that considers autism a 'Western disease'. On the other hand, to fit in the western world, some parents seek a 'normalized' identity; they want their children to fit the British cultural norms (Perepa 2014) and feel under pressure to fit into British society (Munroe et al. 2016).

Another intersection is that parents' gender and gender dynamics varied significantly within families and across communities. Family members do not experience the effects of living with an individual with autism in the same way and have different coping strategies. Mothers paradoxically report both a greater impact on their mental health (depression) and more positive perceptions than fathers (Hastings et al. 2005). The utilization of active coping strategies, emotional support from family members and friends, and acceptance from family members could all lead to lower level of stress and contribute to strong family functioning. Social supports can be made available in online or in community support groups (Zaidman-Zait et al. 2017), in social support networks, and respite care services, and counselling services could support family's coping mechanisms and resilience (Meadan et al. 2010).

An additional dimension to consider is the impact of living in poverty and the income inequality on family experiences of autism. Poverty is found to be a risk factor for emotional problems in children with autism and ADHD (Flouri et al. 2015), and CLD families are more likely to live in poverty. The affordability of care was rarely discussed explicitly in the reviewed papers, but other indirect indicators of living in poverty were mentioned in relation to raising a child with autism, particularly small living conditions and accessing 'nice programmes'. Studies have reported that minorities receive different and less frequent services (Jarquin et al. 2011) and not a large variety of services being unsatisfied with health care services (Zeleke et al. 2019). Social inequalities were a common feature in the experiences of the parents for gaining access to services for early diagnosis and support services. 
A final intersection identified in the papers was the intersection between being CLD and migrant. In 16 out of the 32 studies the participants clearly stated that they are immigrants and in 2 studies migrants. Migrant families might experience more stress due to lack of support from extended family and uncertainties due to immigration status. Furthermore, they may not always know how to navigate the system and how to access support and services due to language barriers and the challenges they face of living in a different culture (Khanlou et al. 2017).

Overall, the data from the scoping review indicate how autism is viewed, managed, and situated within diverse CLD groups. It seems that these issues are complex and perplexed by biological, psychological, cultural, and socio-political factors. Socio-cultural perspectives on disability studies argue that societal structures themselves construct disability and shape participants (Schuelka 2018) and culture has played a key role in how the parents experienced and shaped their understanding of autism. The power of socio-cultural perspectives can be observed in how religion can have a positive impact in some cultures and help the parents cope with having a child with autism (Luong et al. 2009), and furthermore, religion (Munroe et al. 2016) and cultural traditional beliefs can provide models for explaining the cause for autism (Ijalba 2015).

\section{Recommendations for Policy, Research, and Practice}

The current synthesis of the existing literature provides some important points, which can be used to guide policy, research, and practice agendas and recommendations arise from the issues and gaps identified in the literature. Key recommendations include that (1) information to parents, up-to-date materials, and culturally sensitive interventions in multiple languages should be provided; (2) knowledge about the needs of CLD families within the service provision sector and support for service providers should be increased; (3) assessment tools and intervention approaches should take into account cultural and contextual dimensions as well as the autism community views; (4) parents should be involved in diagnosis, assessment, and care with post-diagnostic support; and that (5) researchers should include a diversity of CLD groups in their studies and design studies within the participatory paradigm.

\section{Information to Parents, Up-to-Date Materials, and Culturally Sensitive Interventions in Multiple Languages Should Be Provided}

The reviewed studies showed that parents and CLD communities lack knowledge of autism and this might lead to nonacceptance and stigma within these communities and beyond.
It is vital that policy makers and organizations promote availability of information in multiple languages about autism including information about the uniqueness of bringing up a child with autism to raise awareness and tackle stigma. Embracing positive coping strategies is essential, as is the development of online resources with information about psycho-education and bilingualism to support families with caring responsibilities for children with autism. Parentmediated approaches in different languages can be also useful to practitioners and family members, as well as transparent and up-to-date local information for childcare, respite care, school opportunities, and facilities with autism accommodations can help with access to autism provision services. Practitioners should develop personalized approaches taking into account the cultural background of each community, the family dynamics, and the socioeconomic and educational status of each family in meeting the child's and family's needs.

\section{Knowledge About the Needs of CLD Families Within the Service Provision Sector and Support for Service Providers Should Be Increased}

The findings of the scoping review showed that parents expressed some unmet needs, barriers in navigating the system, and challenges in working with professionals. It seems that these are systemic difficulties because a recent scoping review (Morris et al. 2019) about the experiences of health care providers working with individuals with autism revealed that health care professionals face complexities, which are beyond their professional role and also require access to additional services and resources. In addition, health care providers reported that they experience challenges in communication with autistic people and collaboration with parents and other service providers and that they have limited autism specific knowledge and resources as well as limited autism specific training and prior experience of working with autistic people. These providers also expressed the need for information and training in autism as well as the need for care coordination services and systemic changes so that their working environment becomes autism friendly (Morris et al. 2019). It appears that the need for continuous professional development is paramount as the racial and ethnic disparities in the recognition of autism might be related to the heterogeneity of the presentation of autism and professionals need to be aware of the profile of the children with autism so they can interpret children's behaviour accurately (Mandell et al. 2009) including the subtle cultural differences in the expression of autism in CLD groups. It has been also documented that professionals require training and resources to effectively meet the needs of CLD families (Gardiner and French 2011), and therefore, the provision of continuous professional development of the workforce via online resources could be the starting point for this to achieved. 


\section{Development of Assessment Tools and Intervention Approaches Taking into Account Cultural and Contextual Dimensions As Well as the Autism Community Views}

Further research on assessment tools and intervention approaches with cultural and contextual adaptations taking into account the cultural and contextual dimensions of autism is needed (de Leeuw et al. 2020). The development of culturally appropriate diagnostic and assessment tools is vital for access to effective early intervention or educational support (de Leeuw et al. 2020), and the involvement of parents in assessment sessions can facilitate the interpretation of the child's behaviour from their own cultural perspective. Furthermore, disparities in intervention outcomes among CLD groups may be reduced if interventions are perceived to be more relevant and applicable from the perspective of CLD families (Davenport et al. 2018). This can be achieved by developing an assessment procedure and an intervention, which will be coproduced with parents and other key stakeholders including members of the autism CLD community so that the dimensions of cultural diversity and cultural variation in autism expression could be considered with an impact on improved autism provision.

\section{Parental Involvement in Diagnosis, Assessment, and Care with Post-diagnostic Support}

The cultural and linguistic diversity of families is growing in western countries, and therefore, it is becoming increasingly important to identify ways for supporting individuals with autism, which are sensitive to culture, religion, and language and incorporate the CLD group's values and practices in the approaches used. For this to be achieved, parental participation and involvement in education and care plans are needed as well as involvement in the decision-making process for giving parents the choice among a variety of various intervention approaches, which would be highly valuable for them, and therefore, they will be eager to persevere and attend. Therefore, service providers need to discuss with parents the individual child's needs and the family's needs to elicit family preferences about their willingness for involvement in therapy sessions and for carrying out activities with their children at home, the type of support available, and the type of support the parents might need. In addition, it is recommended that parents' emotional needs are considered throughout the period of diagnosis and intervention and that professionals should be empowered to build positive working relationships with parents (Legg and Tickle 2019). Culturally sensitive information should be provided to families following diagnosis (Munroe et al. 2016), and fathers should be involved in the diagnostic process, so family cohesion and communication are strengthened. Parents could be further supported during diagnosis with the presence of a support coordinator and with a resource support kit.

\section{Researchers to Include a Diversity of CLD Groups and to Design Studies Within the Participatory Paradigm}

A notable absence in the research was the inclusion of diverse CLD groups, and this was more apparent in the research studies published in UK, which included mainly participants of Somalian and Asian background. This might be an area of further investigation in future research considering the wide range of cultural and linguistic communities in the USA and in the UK so more ethnicities will be represented within the CLD groups. In terms of methodological design, studies including a participatory paradigm are overall lacking and future research should engage and include individuals with autism from CLD communities in the research process as co-creators of research and knowledge (Fletcher-Watson et al. 2019) so that their voices are heard and included in the research process with an aim of enhancing their quality of life and the support services. Deepening our understanding of the cultural dimensions of autism may improve the identification, diagnosis, and access to services of families with children with autism from CLD groups.

\section{Strengths and Limitations}

A comprehensive approach was followed by consulting all authors, holding meetings, and conducting reliability checks for papers including in the scoping review and for the coding of the themes. Most of the reviewed studies were based on specific ethnicities, and participants' experiences varied widely depending on culture, gender, community, and location of study. It would thus be simplistic to suggest that the points discussed are universal to all cultural and linguistical diverse groups. However, this is a first attempt to address the topic rather than make general conclusions as there are variations within the autism spectrum and within each group as influenced by the cultural context and the acculturation context (Berry 2006).

The review only included studies published in English and conducted in the USA and in the UK. The reasons for this were to be rigorous, systematic, and clear on the conclusions drawn as the majority of the studies in the autism field are published in the USA and the UK. Another limitation is that the authors did not conduct a quality assessment of the included papers as this was outside the boundaries of conducting a scoping review.

Finally, while the authors did not search specifically for studies with participants from the autism community coming from diverse and cultural and linguistic backgrounds, it is important to note that in the initial search and screening of articles no studies appeared capturing the voice of individuals with autism from such backgrounds and further research is needed in this area to address the existing gap. 


\section{Conclusions}

Given the complex phenomena associated with the experiences of parents from cultural and linguistic diverse backgrounds, this article presents a much-needed scoping review to illustrate what is known about this matter. From the sources reviewed it can be understood that parents' beliefs and experiences are influenced by the medical model of disability, which gives emphasis on diagnosis, symptoms, and stigma; parents request culturally sensitive interventions; and that there are diverse views across different cultures. Sociocultural factors and intersectionality are key constructs, which need to be considered and addressed in future studies. Greater understanding of different cultures, raising awareness about autism in different languages, and further research are needed to understand the barriers CLD groups face and to develop guidelines and support programs, which are culturally sensitive.

Acknowledgments We would like to thank the graduate students Ms. Andreea Laura Cristescu (UK based) and Ms. Stacy Nicole McGuire (USA based) who assisted with the matrix and the inter-rater reliability check.

Funding Information This review was supported by 'BirminghamIllinois Partnership for Discovery, Engagement and Education' ('BRIDGE Seed Fund') agreement award between the University of Birmingham and the University of Illinois at Urbana-Champaign.

\section{Compliance with Ethical Standards}

Conflict of Interest The authors declare that they have no conflict of interest.

Open Access This article is licensed under a Creative Commons Attribution 4.0 International License, which permits use, sharing, adaptation, distribution and reproduction in any medium or format, as long as you give appropriate credit to the original author(s) and the source, provide a link to the Creative Commons licence, and indicate if changes were made. The images or other third party material in this article are included in the article's Creative Commons licence, unless indicated otherwise in a credit line to the material. If material is not included in the article's Creative Commons licence and your intended use is not permitted by statutory regulation or exceeds the permitted use, you will need to obtain permission directly from the copyright holder. To view a copy of this licence, visit http://creativecommons.org/licenses/by/4.0/.

\section{References}

Abbott, M., Bernard, P., \& Forge, J. (2013). Communicating a diagnosis of autism spectrum disorder-a qualitative study of parents' experiences. Clinical Child Psychology and Psychiatry, 18(3), 370-382. https://doi.org/10.1177/1359104512455813.

Angell, A. M., \& Solomon, O. (2017). 'If I was a different ethnicity, would she treat me the same?': Latino parents' experiences obtaining autism services. Disability \& Society, 32(8), 1142-1164. https://doi.org/10.1080/09687599.2017.1339589.
Arksey, H., \& O'Malley, L. (2005). Scoping studies: towards a methodological framework. International Journal of Social Research Methodology, 8(1), 19-32. https://doi.org/10.1080/ 1364557032000119616 .

Bailey Jr., D. B., \& Simeonsson, R. J. (1988). Assessing needs of families with handicapped infants. The Journal of Special Education, 22(1), 117-127. https://doi.org/10.1177/002246698802200113.

Baio, J., Wiggins, L., Christensen, D. L., et al. (2018). Prevalence of autism spectrum disorder among children aged 8 years - autism and developmental disabilities monitoring network, 11 sites, United States, 2014. MMWR Surveillance Summaries, 67(No. SS6), 1-23. https://doi.org/10.15585/mmwr.ss6706a1.

Baird, G., Simonoff, E., Pickles, A., Chandler, S., Loucas, T., Meldrum, D., \& Charman, T. (2006). Prevalence of disorders of the autism spectrum in a population cohort of children in South Thames: the Special Needs and Autism Project (SNAP). The Lancet, 368(9531), 210-215. https://doi.org/10.1016/s0140-6736(06)69041-7.

Baker, D. (2017). The language question: considering three Somali American students with autism. Multiple Voices for Ethnically Diverse Exceptional Learners, 17(1), 20-38. https://doi.org/10. 5555/1547-1888.17.1.20.

Baxter, A. J., Brugha, T. S., Erskine, H. E., Scheurer, R. W., Vos, T., \& Scott, J. G. (2015). The epidemiology and global burden of autism spectrum disorders. Psychological Medicine, 45(3), 601-613. https://doi.org/10.1017/s003329171400172x.

Benevides, T. W., Carretta, H. J., \& Mandell, D. S. (2016). Differences in perceived need for medical, therapeutic, and family support services among children with ASD. Pediatrics, 137, S176-S185. https://doi. org/10.1542/peds.2015-2851p.

Berry, J. W. (2006). Contexts of acculturation. In D. L. Sam \& J. W. Berry (Eds.), The Cambridge handbook of acculturation psychology (pp. 27-42). Cambridge University Press. https://doi.org/10.1017/ CBO9780511489891.006.

Bolton, S., McDonald, D., Curtis, E., Kelly, S., \& Gallagher, L. (2014). Autism in a recently arrived immigrant population. European Journal of Pediatrics, 173(3), 337-343. https://doi.org/10.1007/ s00431-013-2149-6.

Burke, M. M., \& Goldman, S. E. (2018). Special education advocacy among culturally and linguistically diverse families. Journal of Research in Special Educational Needs, 18, 3-14. https://doi.org/ 10.1111/1471-3802.12413.

Burkett, K., Morris, E., Manning-Courtney, P., Anthony, J., \& ShambleyEbron, D. (2015). African American families on autism diagnosis and treatment: the incluence of a culture. Journal of Autism and Developmental Disorders, 45, 3244-3254. https://doi.org/10.1007/ s10803-015-2482-x.

Centers for Disease Control. (2014). Prevalence of autism spectrum disorder among children aged 8 years - autism and developmental disabilities monitoring network, 11 sites, United States, 2010. Morbidity and Mortality Weekly Report, 63(SS2), 1-21.

Cohen, S. R., \& Miguel, J. (2018). Amor and social stigma: ASD beliefs among immigrant Mexican parents. Journal of Autism and Developmental Disorders, 48, 1995-2009. https://doi.org/10.1007/ s10803-017-3457-x.

Crane, L., Chester, J. W., Goddard, L., Henry, L. A., \& Hill, E. (2016). Experiences of autism diagnosis: a survey of over 1000 parents in the United Kingdom. Autism, 20(2), 153-162. https://doi.org/10. 1177/1362361315573636.

Davenport, M., Mazurek, M., Brown, A., \& McCollom, E. (2018). A systematic review of cultural considerations and adaptation of social skills interventions for individuals with autism spectrum disorder. Research in Autism Spectrum Disorders, 52, 23-33. https://doi.org/ 10.1016/j.rasd.2018.05.003.

de Leeuw, A., Happé, F., \& Hoekstra, R. A. (2020). A conceptual framework for understanding the cultural and contextual factors on autism 
across the globe. Autism Research. https://doi.org/10.1002/aur. 2276.

Decoteau, C. L. (2017). The "Western disease": autism and Somali parents' embodied health movements. Social Science \& Medicine, 177, 169-176. https://doi.org/10.1016/j.socscimed.2017.01.064.

Dockrell, J., Lindsay, G., Roulstone, S., \& Law, J. (2014). Supporting children with speech, language and communication needs: an overview of the results of the Better Communication Research Programme. International Journal of Language \& Communication Disorders, 49(5), 543-557. https://doi.org/10.1111/1460-6984. 12089.

DuBay, M., Watson, L. R., \& Zhang, W. (2018). In search of culturally appropriate autism interventions: perspectives of Latino caregivers. Journal of Autism and Developmental Disorders, 48, 1623-1639. https://doi.org/10.1007/s10803-017-3394-8.

Elsabbagh, M., Divan, G., Koh, Y. J., Kim, Y. S., Kauchali, S., Marcín, C., et al. (2012). Global prevalence of autism and other pervasive developmental disorders. Autism Research, 5(3), 160-179. https:// doi.org/10.1002/aur.239

Fletcher-Watson, S., Adams, J., Brook, K., Charman, T., Crane, L., Cusack, J., et al. (2019). Making the future together: shaping autism research through meaningful participation. Autism, 23(4), 943-953. https://doi.org/10.1177/1362361318786721.

Flouri, E., Midouhas, E., Charman, T., \& Sarmadi, Z. (2015). Poverty and the growth of emotional and conduct problems in children with autism with and without comorbid ADHD. Journal of Autism and Developmental Disorders, 45(9), 2928-2938. https://doi.org/10. 1007/s10803-015-2456-z.

Fox, F., Aabe, N., Turner, K., Redwood, S., \& Rai, D. (2017). "It was like walking without knowing where I was going": a qualitative study of autism in a UK Somali migrant community. Journal of Autism and Developmental Disorders, 47, 305-315. https://doi.org/10.1007/ s10803-016-2952-9.

Garcia y, E. F., Breslau, J., Hansen, R., \& Miller, E. (2012). Unintended consequences: an ethnographic narrative case series exploring language recommendations for bilingual families of children with autistic spectrum disorders. Journal of Medical Speech-Language Pathology, 20(2), 10-17.

Gardiner, E., \& French, C. (2011). The relevance of cultural sensitivity in early intervention. Exceptionality Education International, 21(3), 33-49 Retrieved from https://ir.lib.uwo.ca/eei/vol21/iss3/4.

Gilligan, P. (2013). The challenge of cultural explanations and religious requirements for children with autism spectrum conditions: South Asian Muslim parents in Bradford, England. Journal of Religion, Disability \& Health, 17, 393-408. https://doi.org/10.1080/ 15228967.2013.841365.

Gonzalez-Barrero, A. M., \& Nadig, A. (2018). Bilingual children with autism spectrum disorders: the impact of amount of language exposure on vocabulary and morphological skills at school age. Autism Research, 11(12), 1667-1678. https://doi.org/10.1002/aur.2023.

Hampton, S., Rabagliati, H., Sorace, A., \& Fletcher-Watson, S. (2017). Autism and bilingualism: a qualitative interview study of parents' perspectives and experiences. Journal of Speech, Language, and Hearing Research, 60, 435-446. https://doi.org/10.31234/osf.io/ $76 x f s$.

Hannon, M. (2017). Acknowledging intersectionality: aan autoethnography of a Black school counselor educator and father of a student with autism. The Journal of Negro Education, 86(2), 154-162. https://doi.org/10.7709/jnegroeducation.86.2.0154.

Hannon, M. D., Johnson, K. F., Christian, N. A., \& Hannon, L. V. (2018). Black and Latino fathers of students with autism: culturally responsive support. Journal of School Counseling, 15(10), 1-31 https:// digitalcommons.odu.edu/chs_pubs/42.

Hastings, R. P., Kovshoff, H., Brown, T., Ward, N. J., Espinosa, F. D., \& Remington, B. (2005). Coping strategies in mothers and fathers of preschool and school-age children with autism. Autism, 9(4), 377391. https://doi.org/10.1177/1362361305056078.

Hennel, S., Coates, C., Symeonides, C., Gulenc, A., Smith, L., Price, A. M., \& Hiscock, H. (2016). Diagnosing autism: contemporaneous surveys of parent needs and paediatric practice. Journal of Paediatrics and Child Health, 52(5), 506-511. https://doi.org/10. 1111/jpc. 13157.

Hodgetts, S., Zwaigenbaum, L., \& Nicholas, D. (2015). Profile and predictors of service needs for families of children with autism spectrum disorders. Autism, 19(6), 673-683. https://doi.org/10.1177/ 1362361314543531

Hoekstra, R. A., Girma, F., Tekola, B., \& Yenus, Z. (2018). Nothing about us without us: the importance of local collaboration and engagement in the global study of autism. BJPsych International, 15(2), 40-43. https://doi.org/10.1192/bji.2017.26.

Huang, M., \& Zhou, Z. (2016). Perceived self-efficacy, cultural values, and coping styles among Chinese families of children with autism. International Journal of School and Educational Psychology, 4(2), 61-70. https://doi.org/10.1080/21683603.2016.1130562.

Hussein, A. M., Pellicano, E., \& Crane, L. (2019). Understanding and awareness of autism among Somali parents living in the United Kingdom. Autism, 23(6), 1408-1418. https://doi.org/10.1177/ 1362361318813996.

Ijalba, E. (2015). Understanding parental engagement in Hispanic mothers of children with autism spectrum disorder: application of a process-model of cultural competence. Journal of Multilingual Education Research, 6(1), 91-110 https://fordham.bepress.com/ jmer/vol6/iss $1 / 6$.

Ijalba, E. (2016). Hispanic immigrant mothers of young children with autism spectrum disorders: how do they understand and cope with autism? American Journal of Speech-Language Pathology, 25(2), 1-14. https://doi.org/10.1044/2015_AJSLP-13-0017.

Jarquin, V. G., Wiggins, L. D., Schieve, L. A., \& Van Naarden-Braun, K. (2011). Racial disparities in community identification of autism spectrum disorders over time: Metropolitan Atlanta, Georgia, 2000-2006. Journal of Developmental \& Behavioral Pediatrics, 32(3), 179-187. https://doi.org/10.1097/DBP.0b013e31820b4260.

Jegatheesan, B. (2011). Multilingual development in children with autism: Perspectives of South Asian Muslim immigrant parents on raising a child with a communicative disorder in multilingual contexts. Bilingual Research Journal, 34(2), 185-200. https://doi.org/ 10.1080/15235882.2011.597824.

Jegatheesan, B., Fowler, S., \& Miller, P. J. (2010a). From symptom recognition to services: how South Asian Muslim immigrant families navigate autism. Disability \& Society, 25(7), 797-811. https:// doi.org/10.1080/09687599.2010.520894.

Jegatheesan, B., Miller, P. J., \& Fowler, S. A. (2010b). Autism from a religious perspective: a study of parental beliefs in South Asian Muslim immigrant families. Focus on Autism and Other Developmental Disabilities, 25(2), 98-109. https://doi.org/10. 1177/1088357610361344.

Kapp, S. K., Gillespie-Lynch, K., Sherman, L. E., \& Hutman, T. (2013). Deficit, difference, or both? Autism and neurodiversity. Developmental Psychology, 49(1), 59-71. https://doi.org/10.1037/ a0028353.

Khanlou, N., Haque, N., Mustafa, N., Vazquez, L. M., Mantini, A., \& Weiss, J. (2017). Access barriers to services by immigrant mothers of children with autism in Canada. International Journal of Mental Health and Addiction, 15(2), 239-259. https://doi.org/10.1007/ s11469-017-9732-4.

Kim, H. U. (2012). Autism across cultures: rethinking autism. Disability \& Society, 27(4), 535-545. https://doi.org/10.1080/09687599.2012. 659463.

Kim, J., \& Kim, S. (2017). Positioning of Korean immigrant mothers of children with disabilities. International Journal of Multicultural Education, 19(3), 41-64. https://doi.org/10.18251/ijme.v19i3.1362. 
Kim, H. U., \& Roberti, M. (2014). "Tengo Que Habla Español. Yo No Entiendo Ingles!': a qualitative case study on a bilingual child with autism spectrum conditions. Journal of Special Education Apprenticeship, 3(1), n1 http://scholarworks.lib.csusb.edu/josea/ vol3/iss1/7.

Kinnear, S. H., Link, B. G., Ballan, M. S., \& Fischbach, R. L. (2016). Understanding the experience of stigma for parents of children with autism spectrum disorder and the role stigma plays in families' lives. Journal of Autism and Developmental Disorders, 46(3), 942-953. https://doi.org/10.1007/s10803-015-2637-9.

Legg, H., \& Tickle, A. (2019). UK parents' experiences of their child receiving a diagnosis of autism spectrum disorder: a systematic review of the qualitative evidence. Autism, 23(8), 1897-1910. https:// doi.org/10.1177/1362361319841488.

Lehti, V., Hinkka-Yli-Salomäki, S., Cheslack-Postava, K., Gissler, M., Brown, A. S., \& Sourander, A. (2013). The risk of childhood autism among second-generation migrants in Finland: A case- control study. BMC Pediatrics. BMC series. https://doi.org/10.1186/14712431-13-171.

Liasidou, A. (2013). Intersectional understandings of disability and implications for a social justice reform agenda in education policy and practice. Disability \& Society, 28(3), 299-312. https://doi.org/10. 1080/09687599.2012.710012.

Lim, N., O'Reilly, M. F., Sigafoos, J., \& Lancioni, G. E. (2018). Understanding the linguistic needs of diverse individuals with autism spectrum disorder: some comments on the research literature and suggestions for clinicians. Journal of Autism and Developmental Disorders, 48(8), 2890-2895. https://doi.org/10. 1007/s10803-018-3532-y.

Lodder, A., Papadopoulos, C., \& Randhawa, G. (2019). Stigma of living as an autism carer: a brief psycho-social support intervention (SOLACE). Study protocol for a randomised controlled feasibility study. Pilot and Feasibility Studies, 5(1), 34. https://doi.org/10. 1186/s40814-019-0406-9.

Lovelace, T. S., Tamayo, S., \& Robertson, R. E. (2018). Experiences of African American mothers of sons with autism spectrum disorder: lessons for improving service delivery. Education and Training in Autism and Developmental Disabilities, 53(1), 3-16.

Luong, J., Yoder, M. K., \& Canham, D. (2009). Southeast Asian parents raising a child with autism: a qualitative investigation of coping styles. The Journal of School Nursing, 25(3), 222-229. https://doi. org/10.1177/1059840509334365.

Magaña, S., Lopez, K., Aguinaga, A., \& Morton, H. (2013). Access to diagnosis and treatment services among Latino children with autism spectrum disorders. Intellectual and Developmental Disabilities, 51(3), 141-153. https://doi.org/10.1352/1934-9556-51.3.141.

Mandell, D. S., Listerud, J., Levy, S. E., \& Pinto-Martin, J. A. (2002). Race differences in the age at diagnosis among Medicaid-eligible children with autism. Journal of the American Academy of Child \& Adolescent Psychiatry, 4l(12), 1447-1453. https://doi.org/10.1097/ 00004583-200212000-00016.

Mandell, D. S., Wiggins, L. D., Carpenter, L. A., Daniels, J., DiGuiseppi, C., Durkin, M. S., Giarelli, E., et al. (2009). Racial/ethnic disparities in the identification of children with autism spectrum disorders. American Journal of Public Health, 99(3), 493-498. https://doi. org/10.2105/ajph.2007.131243.

Meadan, H., Halle, J. W., \& Ebata, A. T. (2010). Families with children who have autism spectrum disorders: stress and support. Exceptional Children, 77(1), 7-36. https://doi.org/10.1177/ 001440291007700101

Moh, T. A., \& Magiati, I. (2012). Factors associated with parental stress and satisfaction during the process of diagnosis of children with autism spectrum disorders. Research in Autism Spectrum Disorders, 6(1), 293-303. https://doi.org/10.1016/j.rasd.2011.05. 011 .
Moher, D., Liberati, A., Tetzlaff, J., the PRISMA Group, et al. (2009). Preferred reporting items for systematic reviews and meta-analyses: the PRISMA statement. Annals of Internal Medicine, 151, 264-269. https://doi.org/10.7326/0003-4819-151-4-200908180-00135.

Morris, R., Greenblatt, A., \& Saini, M. (2019). Healthcare providers' experiences with autism: a scoping review. Journal of Autism and Developmental Disorders, 49(6), 2374-2388. https://doi.org/10. 1007/s10803-019-03912-6.

Munroe, K., Hammond, L., \& Cole, S. (2016). The experiences of African immigrant mothers living in the United Kingdom with a child diagnosed with an autism spectrum disorder: an interpretive phenomenological analysis. Disability \& Society, 31(6), 798-819. https://doi.org/10.1080/09687599.2016.1200015.

Papadopoulos, C., Lodder, A., Constantinou, G., \& Randhawa, G. (2019). Systematic review of the relationship between autism stigma and informal caregiver mental health. Journal of Autism and Developmental Disorders, 49(4), 1665-1685. https://doi.org/10. 1007/s10803-018-3835-Z.

Pearson, J. N., \& Meadan, H. (2018). African American parents' perceptions of diagnosis and services for children with autism. Education and Training in Autism and Developmental Disabilities, 53(1), 1732.

Perepa, P. (2014). Cultural basis of social 'deficits' in autism spectrum disorders. European Journal of Special Needs Education, 29(3), 313-326. https://doi.org/10.1080/08856257.2014.908024.

Ratto, A. B., Reznick, J. S., \& Turner-Brown, L. (2016). Cultural effects on the diagnosis of autism spectrum disorder among Latinos. Focus on Autism and Other Developmental Disabilities, 31(4), 275-283. https://doi.org/10.1177/1088357615587501.

Ravindran, N., \& Myers, B. J. (2012). Cultural influences on perceptions of health, illness, and disability: a review and focus on autism. Journal of Child and Family Studies, 21(2), 311-319. https://doi. org/10.1007/s10826-011-9477-9.

Rollins, P. R., John, S., Jones, A., \& De Froy, A. (2019). Pathways early ASD intervention as a moderator of parenting stress on parenting behaviors: a randomized control trial. Journal of Autism and Developmental Disorders, 49(10), 4280-4293. https://doi.org/10. 1007/s10803-019-04144-4.

Sage, K. D., \& Jegatheesan, B. (2010). Perceptions of siblings with autism and relationships with them: European American and Asian American siblings draw and tell. Journal of Intellectual and Developmental Disability, 35(2), 92-103. https://doi.org/10.3109/ 13668251003712788.

Schuelka, M. J. (2018). The cultural production of the "disabled" person: constructing difference in Bhutanese schools. Anthropology \& Education Quarterly, 49(2), 183-200. https://doi.org/10.1111/aeq. 12244.

Selman, L. E., Fox, F., Aabe, N., Turner, K., Rai, D., \& Redwood, S. (2018). "You are labelled by your children's disability"-a community-based, participatory study of stigma among Somali parents of children with autism living in the United Kingdom. Ethnicity \& Health, 23(7), 781-796. https://doi.org/10.1080/13557858.2017. 1294663.

Thomas, G., \& Macnab, N. (2019). Intersectionality, diversity, community and inclusion: untangling the knots. International Journal of Inclusive Education, 1-18. https://doi.org/10.1080/13603116.2019. 1645892.

Valicenti-McDermott, M., Tarshis, N., Schouls, M., Galdston, M., Hottinger, K., Seijo, R., et al. (2012). Language differences between monolingual English and bilingual English-Spanish young children with autism spectrum disorders. Journal of Child Neurology, 28(7), 945-948. https://doi.org/10.1177/0883073812453204.

Wang, H. T., \& West, E. A. (2016). Asian American immigrant parents supporting children with autism: perceptions of fathers and mothers. International Journal of Whole Schooling, 12(1), 1-21. 
You, H. K., \& Rosenkoetter, S. E. (2014). Listening to Korean immigrant mothers: meanings of raising a child with a disability. Multiple Voices for Ethnically Diverse Exceptional Learners, 14(1), 30-41.

$\mathrm{Yu}, \mathrm{B}$. (2013). Issues in bilingualism and heritage language maintenance: perspectives of minority-language mothers of children with autism spectrum disorders. American Journal of Speech-Language Pathology, 22(1), 10-24. https://doi.org/10.1044/10580360(2012/10-0078.

$\mathrm{Yu}, \mathrm{B}$. (2016). Bilingualism as conceptualized and bilingualism as lived: a critical examination of the monolingual socialization of a child with autism in a bilingual family. Journal of Autism and Developmental Disorders, 46(2), 424-435. https://doi.org/10. 1007/s10803-015-2625-0.

Zaidman-Zait, A., Mirenda, P., Duku, E., Vaillancourt, T., Smith, I. M., Szatmari, P., et al. (2017). Impact of personal and social resources on parenting stress in mothers of children with autism spectrum disorder. Autism, 21(2), 155-166. https://doi.org/10.1177/ 1362361316633033.

Zechella, A. N., \& Raval, V. V. (2016). Parenting children with intellectual and developmental disabilities in Asian Indian families in the United States. Journal of Child and Family Studies, 25(4), 12951309. https://doi.org/10.1007/s10826-015-0285-5.

Zeleke, W. A., Hughes, T. L., \& Drozda, N. (2019). Disparities in diagnosis and service access for minority children with ASD in the United States. Journal of Autism and Developmental Disorders, 49, 4320-4331. https://doi.org/10.1007/s10803-019-04131-9.

Publisher's Note Springer Nature remains neutral with regard to jurisdictional claims in published maps and institutional affiliations. 